\title{
Transmission Efficiency of Cucumber mosaic virus by Aphids Associated with Virus Epidemics in Snap Bean
}

\author{
F. E. Gildow, D. A. Shah, W. M. Sackett, T. Butzler, B. A. Nault, and S. J. Fleischer
}

First and third authors: Department of Plant Pathology, Pennsylvania State University, University Park, PA 16802; second and fifth authors: Department of Plant Pathology and Plant-Microbe Biology and Department of Entomology, respectively, New York State Agricultural Experiment Station, Geneva, NY 14456; fourth author: Cooperative Extension, Pennsylvania State University, University Park, PA 16802; and sixth author: Department of Entomology, Pennsylvania State University, University Park, PA 16802.

Accepted for publication 22 July 2008.

\begin{abstract}
Gildow, F. E., Shah, D. A., Sackett, W. M., Butzler, T., Nault, B. A., and Fleischer, S. J. 2008. Transmission efficiency of Cucumber mosaic virus by aphids associated with virus epidemics in snap bean. Phytopathology 98:1233-1241.

Cucumber mosaic virus (CMV) is a major component of the virus complex that has become more pronounced in snap bean in the midwestern and northeastern United States since 2001. Multiple-vectortransfer tests were done to estimate the CMV transmission efficiencies $(p)$ of the main aphid species identified in commercial snap bean fields in

A. spiraecola, A. craccivora, Macrosiphum euphorbiae, and Rhopalosiphum maidis. Poor vectors $(\hat{p}<0.01)$ included A. fabae, Nearctaphis bakeri, and Myzus persicae. Only one species, Sitobion avenae, failed to transmit CMV in replicated tests. Estimates of $p$ were consistent between different clones of the same aphid species and among three different field isolates of CMV tested. Single-vector-transfer test results for a subset of the species supported those obtained via the multiple-vector-transfer approach. Our results are consistent with the notion that A. glycines is a major vector of recent CMV epidemics in snap bean, but that species is only one of several that are involved.
\end{abstract} New York and Pennsylvania. The four most efficient vectors $(\hat{p}>0.05)$ were Aphis gossypii, A. glycines, Acyrthosiphon pisum, and Therioaphis trifolii, which were all significant species in the migratory aphid populations in snap bean. Moderately efficient vectors $(0.01<\hat{p}<0.04)$ were
Additional keywords: empirical Bayesian estimator, group testing, multiple comparisons, soybean aphid, vector-transfer design.
Cucumber mosaic virus (CMV) is one of the most common virus pathogens of plants, and infects over 1,000 species (27). CMV, type member of the genus Cucumovirus in the family Bromoviridae (22), is a multicomponent virus consisting of three genomic single-stranded RNA's each encapsidated individually in a 28-nm diameter icosahedral particle. CMV is aphid transmitted in a stylet-borne nonpersistent manner (21). The virus capsid protein interacts directly with components of the aphid stylet to influence transmission efficiency $(7,23)$, and does not utilize a virus-encoded helper component protein, as do nonpersistently transmitted potyviruses.

Many strains of CMV have been described differing in host range, symptoms, and aphid vectors, in serological properties of structural proteins, and in nucleic acid sequence (22). Most CMV strains are currently divided between two major groups (subgroup 1 and subgroup 2) based on serological and sequence similarities $(1,15)$. Common strains of CMV typically infect solanaceous and cucurbit crops, but not legume species. Legume-infecting strains of CMV isolated from infected peas (Pisum sativum L.) were first described by Whipple and Walker (39). These strains were not seed transmitted, but were transmissible by the green peach aphid (Myzus persicae L.).

Pod yield and quality reducing epidemics of legume-infecting strains of CMV have been reported in snap bean (Phaseolus vulgaris L.) with increasing frequency in New York and several Midwestern states $(18,28,29)$. Similar concerns existed in Pennsylvania. CMV infection of snap bean typically results in severe

Corresponding author: F. E. Gildow; E-mail address: feg2@psu.edu

doi:10.1094/PHYTO-98-11-1233

(c) 2008 The American Phytopathological Society foliar mosaic symptoms in leaves, which were developing at the time of infection, and deformed pods in plants infected at flowering. Yield reductions can be significant and little or no genetic resistance to CMV infection is currently available in commercial snap bean cultivars $(28,32)$.

The widespread CMV epidemics in snap bean from 2000 to 2003 coincided with the detection of the newly introduced soybean aphid, Aphis glycines (Matsumura) (26). This led to speculation that the soybean aphid may be responsible for the increased spread of CMV in snap bean. However, the species composition of aphid populations in snap bean fields associated with CMV epidemics and the vector efficiency of different aphid species for legume-infecting strains of CMV were not well known. The soybean aphid is capable of transmitting several legume-infecting viruses in soybean (Glycine max [L.] Merr.) $(8,38)$; however, its role in CMV spread in snap bean was uncertain. In addition, Nault et al. (20) indicated that the predominant aphid species emigrating into snap bean fields is fluid over time and that several other aphid species, including the pea aphid, Acyrthosiphon pisum (Harris), the corn leaf aphid, Rhopalosiphum maidis (Fitch), and the yellow clover aphid, Therioaphis trifolii (Monell) predominated in snap bean fields at different times in different years. The potential role of these aphid species in legume-CMV epidemics was unknown.

Observation of CMV epidemics occurring in snap bean crops prior to the introduction of A. glycines suggested the possible involvement of multiple aphid species. Provvidenti (25) observed similar CMV epidemics in late-planted snap bean fields near Geneva, NY, in 1974. Results of his greenhouse studies with a field-collected strain of CMV from snap bean (CMV-B) verified aphid transmission by the green peach aphid, Myzus persicae (Sulzer), whereas seed transmission was minimal with only one 
of 1,294 seedlings infected when grown from seed harvested from infected plants. Although the severe foliar mosaic symptoms faded in most snap bean cultivars following the "shock phase" of early infection, none of the 195 cultivars and breeding lines tested was resistant to pod distortion following infection at flowering.

In order to develop better-targeted control strategies more information was needed on the mode of CMV spread. Surveys in New York (20) and Pennsylvania (12) identified several dominant aphid species migrating into snap bean fields. However, the ability of these populations of aphids to transmit CMV to snap bean was not known. In this study we identify aphid species most effective in transmitting CMV to snap bean among those that were dominant in the earlier surveys. We also describe a standardized protocol for testing CMV transmission using multiplevector-transfer tests (group testing) to calculate transmission efficiency defined as the probability of a single aphid transmitting the virus (11). Estimates of transmission efficiencies derived from multiple-vector-transfer tests were verified by actual single-aphidtransfer tests. Based on the species composition of alate aphid populations migrating into snap bean crops and on transmission efficiency, A. glycines, A. pisum, R. maidis, and Therioaphis trifolii are all competent vectors that may participate in CMV epidemics. This paper expands a preliminary report made previously (12).

\section{MATERIALS AND METHODS}

Aphid identification. Aphid species frequently found in snap bean fields in New York state have recently been reported (20). To identify migrant aphid species common to snap bean fields in Pennsylvania, aphid trapping was conducted in Centre and Clinton counties during the summer of 2004. Winged aphids were collected weekly during July and August in six late-planted, commercial snap bean fields using five water pan traps per field, each containing a green mottled tile submerged in $20 \%$ propylene glycol, as previously described (20). Fields were divided into quarters with one trap centered in each quarter area and one trap placed in the center of the field. Most fields were bordered by soybean, alfalfa, or maize. Collected aphids were stored in $70 \%$ ethanol until cleared and mounted on slides as previously described (37). Aphids were identified to species according to Smith et al. (30). Verification of species identification was done by $\mathrm{R}$. Eckel (RVWE Consulting, Frenchtown, NJ). Voucher specimens are stored at the Frost Entomological Museum (Department of Entomology, Pennsylvania State University, University Park, PA).

Aphid clone colony maintenance. Once aphids were identified, virus-free colonies of aphid species commonly trapped in the snap bean fields were collected from plants and maintained in the laboratory on a variety of host plants at $22^{\circ} \mathrm{C}$ and $60 \%$ relative humidity under a constant $24 \mathrm{~h}$ photoperiod. Healthy colonies of asexually reproducing clones of each species were initiated using single nonviruliferous, apterous adults. Additionally, a few species or clones within a species were obtained from collaborators in Maryland (MD) or New York (NY) and reared in parallel. Unless otherwise indicated, aphid clones represent aphids collected in Pennsylvania (PA). The aphid species used and the host plant species used for aphid colony maintenance of each species were: pea aphid, Acyrthosiphon pisum (Harris), on fava bean, Vicia faba L.; cowpea aphid, Aphis craccivora Koch, on V. faba; black bean aphid, Aphis fabae Scopoli, on V. faba; soybean aphid, A. glycines Matsumura, on soybean, Glycine max (L.) Merr.; melon or cotton aphid, Aphis gossypii Glover, on cucumber, Cucumis sativus L.; spirea aphid, Aphis spiraecola Patch (= A. citricola van der Goot), on carrot, Daucus carota L.; potato aphid, Macrosiphum euphorbiae (Thomas) on green pepper, Capsicum annuum L.; green peach aphid, Myzus persicae (Sulzer) on turnip, Brassica rapa L.; short-beaked clover aphid, Nearctaphis bakeri (Cowen), on red clover, Trifolium pratens L.; corn leaf aphid, Rhopalosiphum maidis (Fitch) on barley, Hordeum vulgare L.; bird cherry-oat aphid, Rhopalosiphum padi (L.)., on H. vulgare; English grain aphid, Sitobion avenae (F.), on H. vulgare; yellow clover aphid, Therioaphis trifolii (Monell), on alfalfa, Medicago sativus L. subsp. sativa

CMV isolate sources and maintenance. Three field isolates of CMV obtained from A. Taylor (NYSAES, Geneva, NY) were initially tested for aphid transmission and symptom expression in snap bean. The CMV-Le isolate was originally obtained from C. Grau (University of Wisconsin, Madison, WI) in 2001, and is currently used for snap bean breeding and selection trials at Cornell University; the CMV-Hy isolate was recovered in 2004 from an infected snap bean cv. Hystyle plant at Geneva, NY; and the CMV-B isolate was previously recovered from infected snap bean by R. Provvidenti (25). Mosaic symptoms on 'Hystyle' following mechanical or aphid inoculations by these isolates were similar. All isolates were initially maintained by a series of standard mechanical inoculations using infected snap bean leaf homogenate in $0.1 \mathrm{M}$ phosphate buffer $(\mathrm{pH} 7)$ and Celite abrasive on 10-day-old highly susceptible snap bean seedlings (cv. Hystyle). Severe symptoms of leaf distortion and mosaic appeared within 14 days postinoculation. Following these initial mechanical inoculations, all isolates were further maintained in 'Hystyle' bean using only aphid transmissions by A. gossypii or A. glycines to ensure aphid transmissibility. For routine maintenance transmissions, approximately 50 aphids were allowed to settle for $15 \mathrm{~min}$ on a CMV-infected leaflet showing severe CMV symptoms and then the excised leaflet with aphids placed over a 10-day-old 'Hystyle' bean seedling and caged overnight. The next day the seedling was sprayed with systemic Orthene (acephate; Monsanto Co., San Ramon, CA) insecticide to kill the aphids and $24 \mathrm{~h}$ later the seedling placed in the greenhouse for observation. Typically, all plants infested with aphids became infected by this method. All virus source plants and virus transmission test plants were maintained in an aphid-free limited access air-conditioned greenhouse (Department of Plant Pathology, Pennsylvania State University) with supplemented halogen lighting for a $16 \mathrm{~h}$ photoperiod. All plants were grown in artificial soil mix supplemented with Osmicote slow release fertilizer and weekly fertilization. Plants were maintained on drip irrigation.

Transmission test protocols. To provide equivalent CMVinfected snap bean tissues for virus acquisition from week to week, snap bean plants were inoculated with CMV using viruliferous aphids and the plants then were selected as virus acquisition sources approximately 4 weeks postinoculation. Only the youngest fully expanded leaves displaying severe mosaic and distortion symptoms were used as virus sources. These were usually systemically infected first and second set of trifoliate leaves developing after aphid inoculation of the primary leaves on the young seedling. To initiate virus acquisition probes, aphids were removed from their normal host plant species and placed in plastic dishes with tight fitting lids and starved for $1 \mathrm{~h}$. Symptomatic CMV-infected leaflets were then placed into dishes and aphids were allowed to settle on the leaflet surface and begin probing. Following a 3 to $5 \mathrm{~min}$ probing access period, during which the aphids were observed for probing behavior using a stereo dissecting microscope, the aphids were transferred to 10-day-old healthy snap bean seedlings (cv. Hystyle) by either single vector transfer or multiple vector transfer, which will be described later. The seedling was immediately caged and observed to verify that probing aphids remained on the test seedling over 30 to $60 \mathrm{~min}$. During this time aphids usually made their first test probes on the test seedlings. The caged plants were then placed in a controlled environment room $\left(22^{\circ} \mathrm{C}, 24 \mathrm{~h}\right.$ continuous light photoperiod) overnight. The following day the seedlings were placed in a vented fumigation chamber and sprayed with acephate or pymetrozine (Endeavor, Syngenta Crop Protection, Greensboro, NC) insecticide to kill all aphids. The next day the 
seedlings were moved to the greenhouse where they were observed for CMV symptom development over the next 4 weeks. For all negative control treatments, aphids of the same species were allowed to acquisition-probe on healthy snap bean leaves and were then transferred to healthy snap bean seedlings in parallel treatments. Nonviruliferous stock colony aphids were never observed to transmit CMV or any virus to snap bean during these experiments. For a positive control treatment in each experiment, one treatment always consisted of one of the two efficient vectors, A. gossypii or A. glycines, to verify quality of the CMV source tissue and experimental methods. Failure of either of these aphids to transmit CMV would signal a problem in the protocol or virus sources. These aphid species transmitted as expected in all experiments, indicating consistent CMV titers in source tissues and consistency in transmission parameters. Prior to use, healthy test seedlings were grown in a virus-free and aphid-free, airconditioned greenhouse room and then maintained aphid-free following the aphid inoculation protocol. Replicated tests failed to detect any mechanical inoculation of CMV by physical manipulation during handling protocols or by touching when infected plants were purposely placed into the center of crowded planting trays surrounded by healthy seedlings for up to 5 weeks. In two preliminary tests of protocol, none of the 30 seedlings became infected when overlaid with leaflets from infected plants (data not shown). In addition, infected plants never occurred as nearest neighbor pairs on the greenhouse bench in sparsely infected experiments with scattered infected plants. These observations suggested that our CMV strains were not easily spread by casual contacts. In addition, healthy control plants and healthy seedlings kept in close proximity to infected plants never became infected over a 3-year period of testing. Therefore, all infected seedlings were presumed to result only from aphid transmission.

Multiple-vector-transfers (MVT). For MVT (group) testing, 10 aphids were placed on snap bean leaf tissue infected with CMV-Le, observed to initiate virus acquisition probing over a 3 to 5 min period, after which the leaflet piece with all 10 probing aphids was placed on a 10-day-old snap bean seedling (cv. Hystyle or Zeus) for a $16 \mathrm{~h}$ inoculation period. The next day, the seedlings were observed to verify that all aphids had moved to the test seedling, and then the seedlings were sprayed with acephate, placed in a greenhouse, and observed for CMV symptoms as described previously. For each aphid species treatment, 10 seedlings were infested with 10 aphids each for a total of 50 seedlings per clone over five replicated experiments. Negative controls consisted of aphids of each clone allowed to probe only on healthy plants in parallel treatments.

Single-vector transfers (SVT). We next compared CMV-Le transmission by individual aphids of five aphid clones representing four species, A. glycines (NY and PA clones), A. gossypii (NY), M. persicae (PA), and R. maidis (PA). Based on previously completed MVT tests, these species ranged from efficient to poor vectors. Individual aphids were observed to probe on an infected snap bean leaf for about $3 \mathrm{~min}$, then one aphid was transferred individually to a single healthy 10-day-old 'Hystyle' seedling using a camel hair brush. The individual aphids were observed to verify that they stayed on plants and commenced probing, after which the plants were caged for a $16 \mathrm{~h}$ inoculation period. For each treatment (clone), 120 aphids were placed individually on 120 seedlings. Each treatment was independently replicated three times. The plants were then sprayed and treated as described previously. Negative controls consisted of aphids of each clone fed on healthy leaves in parallel treatments.

Comparison of legume-infecting CMV isolates. A. glycines (PA clone) and $M$. persicae (PA clone) were tested for their ability to transmit three isolates of CMV (CMV-B, CMV-Le, and CMVHy) obtained from different source locations or times. All three isolates were originally isolated from snap bean and previously had been maintained in snap bean by sequential mechanical inoculations. Additionally, CMV-B had been stored several years at $-80^{\circ} \mathrm{C}$ before recovery by mechanical inoculation to snap bean. All isolates were transferred to snap bean and maintained through at least two sequential $M$. persicae transmissions before use. CMV transmission efficiencies were determined through MVT tests, as described previously, 10 aphids per plants and 45 plants tested per isolate-aphid clone combination in three replicated experiments. Positive and negative controls also consisted of 45 plants each, but group size was increased from 10 to 50 aphids per plant. All test seedlings were enzyme-linked immunosorbent assay (ELISA)-tested as described later to confirm infection.

ELISA testing. All seedlings used in the MVT and SVT transmission tests were ELISA-tested to verify CMV infection using commercial detection kits and associated protocols (Agdia Inc., Elkhart, IN). All test seedlings were initially scored for symptom production at 4 weeks postinoculation and then samples consisting of two leaflets were selected for ELISA testing. Leaves were placed in numbered, plastic mesh sample bags and homogenized in $5 \mathrm{ml}$ of sample buffer with a ball-bearing extractor (Agdia Inc.) and drill press. All samples were tested in paired wells. Healthy controls consisted of noninoculated symptomless snap bean leaf tissue from 21-day-old seedlings. Positive controls consisted of mature leaf tissue showing severe mosaic and distortion symptoms from chronically infected stock snap bean plants (cv. Hystyle). In addition, the uninfected negative control treatments (plants fed on by nonviruliferous aphids allowed to probe only on healthy tissue) and the positive control treatments (plants inoculated with CMV by A. glycines or A. gossypii, to verify infectivity of source plants) were always ELISA-tested together with the experimental treatments.

Statistical analysis-MVT. It is well known that MVT tests (also called group testing) can be more economical than SVT tests when attempting to estimate transmission probabilities that are relatively low $(31,35)$. With the MVT design, groups of $s$ aphids are allowed to acquire the virus by feeding on virusinfected plant material, and the entire group (size $s$ ) is transferred to a healthy plant. The process is repeated for each of $n$ healthy plants. Of course, if $s=1$, then we have the SVT design. Let $t$ be the number of plants out of $n$ which subsequently become infected by the virus. Given $s, t$, and $n$, the interest is in obtaining estimates and confidence intervals for $p_{i}$, the probability of virus transmission by a single aphid of clone $i$. Because soybean aphid is a new component of the aphid complex occurring in snap bean fields, another goal was to obtain estimates of the difference in CMV transmission efficiency between aphid clones and the $A$. glycines (PA) clone. We used an empirical Bayesian approach for obtaining point estimates of $p_{i}$ with the MVT data, but first briefly describe the more familiar maximum likelihood (i.e., frequentist) estimation method.

Assume that $t$ has a binomial distribution (we return to this assumption in more detail in the Discussion) with parameters $n$ and $\theta$ (i.e., $T \approx \operatorname{Bin}[n, \theta]$ ). Dropping the subscript $i$ for clarity, it is straightforward to show that $\theta=(1-p)^{s}(31,33)$. The maximum likelihood estimator for $p$ (i.e., $\hat{p}_{\mathrm{MLE}}$ ) is given by

$$
\hat{p}_{\mathrm{MLE}}=1-(1-\hat{\theta})^{1 / \mathrm{s}}
$$

where $\hat{\theta}=t / n$.

The estimator $\hat{p}_{\mathrm{MLE}}$ is positively biased for all $n<\infty(16,31)$. The Burrows estimator (6),

$$
\hat{p}_{\mathrm{B}}=1-\left[1-\frac{t}{n+b}\right]^{1 / s}
$$

where $b=(s-1) / 2 s$, is an improvement as it partially corrects for the bias. We next describe a more recent empirical Bayesian estimator which has lower bias compared with $\hat{p}_{\mathrm{MLE}}$ and, for $n$ and $s$ used in our study, a smaller Bayes risk compared with $\hat{p}_{\mathrm{B}}(2)$. 
MVT tests: Point estimates (empirical Bayesian approach). The approach is briefly outlined here. For theoretical and computational details, see Bilder and Tebbs (2) and Tebbs et al. (34). The method hinges on specifying a one-parameter beta family prior distribution $f_{p}\left(p \mid \beta=\beta(1-p)^{\beta-1}, 0<p<1\right.$ for $p$ (34). The marginal distribution of $T$

$$
f_{T}(t \mid \beta)=\frac{\beta \Gamma(n+1) \Gamma\left(n-t+\frac{\beta}{s}\right)}{s \Gamma(n-t+1) \Gamma\left(n+\frac{\beta}{s}+1\right)}
$$

is used to elicit an estimate of $\beta$ (2). We used the marginal method of moments estimate of $\beta$

$$
\hat{\beta}_{\mathrm{MOM}}=\frac{s(1-\hat{\theta})}{\hat{\theta}}
$$

as a starting value in the maximization of $\log \left(f_{T}(t \mid \beta)\right.$ to find the maximum likelihood estimate (MLE) of $\beta\left(\hat{\beta}_{\mathrm{MLE}}\right)$. The estimate $\hat{\beta}_{\mathrm{MLE}}$ is used in place of $\beta$ in the estimator

$$
\hat{p}_{\mathrm{EB}}=1-\left[1-\frac{t+1}{n+\frac{\beta}{s}+1}\right]^{1 / s}
$$

to arrive at the empirical Bayes estimate of $p$.

When $t=0$, as for the $S$. avenae (NY) clone data, $\hat{\beta}_{\mathrm{MLE}}$ cannot be computed (2). As a workaround when $t=0$, we used $t=t+0.5$ to facilitate the computations.

MVT tests: Credible intervals (empirical Bayesian approach). With the frequentist approach, one can obtain confidence intervals. The empirical Bayesian approach leads to credible intervals, which differ in concept from confidence intervals. We briefly revisit the two concepts. A 95\% confidence interval for a parameter (in this case $p$ ) means that if the experiment was repeated numerous times, and a confidence interval constructed from each data set, then $95 \%$ of the intervals will contain $p$. In the Bayesian framework, a 95\% credible interval $(l$ and $u$ ) would be interpreted to mean that there is a $95 \%$ posterior probability $p$ is in the interval defined by the limits $l$ and $u$.

Details are again found in Bilder and Tebbs (2). The posterior distribution of $P$ given $T=t$ is given by

$$
f_{P \mid T}(p \mid t, \beta)=\frac{s \Gamma\left(n+\frac{\beta}{s}+1\right)}{\Gamma\left(n-t+\frac{\beta}{s}\right) \Gamma(t+1)}(1-p)^{s(n-t)+\beta-1}\left[1-(1-p)^{s}\right]^{t}
$$

The empirical pQsterior distribution is found by substituting the data for $s, n$, and $\beta_{\mathrm{MLE}}$ for $\beta$ into equation 4 . For the MVT data, plots of $f_{P \mid T}(p \mid t, \beta)$ versus $p$ show that the distribution is unimodal and skewed (data not shown). When the empirical distribution of $f_{P \mid T}(p \mid t, \beta)$ has these properties, it may be more appropriate to use the highest posterior density (HPD) credible interval rather than the equal-tail credible interval (2). In order to obtain the HPD interval, one has to solve the following system of equations for $a$ and $b$

$$
\left.\begin{array}{l}
f_{P \mid T}(a \mid \text { data })=f_{P \mid T}(b \mid \text { data }) \\
\int_{a}^{b} f_{P \mid T}(p \mid \text { data }) d p=1-\alpha
\end{array}\right\}
$$

where data represents the empirical values of $s, n, t$, and $\hat{\beta}_{\mathrm{MLE}}$. The value of $\alpha$ determines the percent credibility, so $\alpha=0.05$ would represent a $95 \%$ interval. When equation 5 is solved for $a$ and $b$, one then has a $(1-\alpha)$ percent HPD credible interval for $p$ (36).
MVT tests: Pairwise comparisons. Two sided Dunnett (10) simultaneous $95 \%$ confidence intervals for $\psi_{i}=\left(p_{i}-p_{0}\right)$ were constructed, where $p_{i}$ is the probability of CMV transmission by a single aphid of clone $i$, and $p_{0}$ is the transmission probability for A. glycines (PA). Using $\hat{p}_{\mathrm{MLE}}$ as the estimate of $p$, simultaneous confidence interval for $\psi_{i}$ are given by

$$
\hat{\Psi}_{i} \pm q_{\alpha, k, \infty} \sqrt{\left(\hat{u}_{i} / n\right)_{i}+\left(\hat{u}_{0} / n_{0}\right)}
$$

where

$$
\hat{u}_{y}=s_{y}^{-2}\left\{1-\left(1-\hat{p}_{y}\right)^{s_{y}}\right\}\left(1-\hat{p}_{y}\right)^{2-s_{y}}
$$

$k$ is the number of comparisons being made simultaneously against $p_{0}$, and $q_{\alpha, k, \infty}$ is the critical value from Dunnett's twosided range distribution (19).

SVT. SVT tests were done with A. glycines (NY), A. glycines (PA), A. gossypii (NY), M. persicae (PA), and R. maidis (PA), thus representing five different clones. Let $x$ be the number of healthy test seedlings that became infected with CMV out of $n$ seedlings on which individual viruliferous aphids were allowed to probe (one per plant). Then a point estimate $\left(\hat{p}_{i}\right)$ of the probability of CMV transmission by a single aphid of clone $i\left(p_{i}\right)$ is given by $x_{\mathrm{i}} / n_{\mathrm{i}}$. One can also calculate a confidence interval for $p_{\mathrm{i}}$. Although the Wald interval is widely familiar, it is problematic in terms of coverage probability (4). A superior alternative, which we use here, is the Agresti-Coull interval. Let $\kappa=\Phi^{-1}(1-\alpha / 2)$, where $\Phi(z)$ is the standard normal cumulative distribution, and $\alpha$ is between 0 and 1. For a 95\% confidence interval, $\alpha=0.05$. Following (4), let $\tilde{x}=x+\left(\kappa^{2} / 2\right), \tilde{n}=n+\kappa^{2}, \quad \tilde{p}=\tilde{x} / \tilde{n}$, and $\widetilde{q}=1-\tilde{p}$. Then the Agresti-Coull interval is given by

$$
\tilde{p}_{i} \pm \kappa(\tilde{p} \tilde{q})^{1 / 2} \tilde{n}^{-1 / 2}
$$

Because soybean aphid is a recently introduced species and has been implicated as a major player in the outbreaks of virus epidemics in the Midwest and Northeast (18,32), we were interested in how CMV transmission efficiency of A. glycines compared with that of other species found in the region prior to the soybean aphid's introduction. Let $p_{0}$ be the transmission efficiency (probability) for the A. glycines (PA) clone. Interest is in the difference $\delta_{i}=\left(p_{i}-p_{0}\right), i=1, \ldots, \mathrm{M}$, corresponding to the other clones tested (in the present context $\mathrm{M}=4$ ). Therefore, $\delta_{i}$ represents the difference in CMV transmission efficiency of clone $i$ compared with that of $A$. glycines (PA). A $95 \%$ confidence interval for $\delta_{i}$ is given by

$$
\hat{\delta}_{i} \pm \mathrm{z}_{\alpha, \mathrm{M}, \Sigma}\left(\hat{v}_{i}+\hat{v}_{0}\right)^{1 / 2}
$$

where $\hat{v}_{0}=\hat{p}_{0}\left(1-\hat{p}_{0}\right) / n_{0}, \hat{v}_{i}=\hat{p}_{i}\left(1-\hat{p}_{i}\right) / n_{i}$, and $z_{\alpha, M, \Sigma}$ is the $\alpha$ level critical value for a M-variate standard normal with correlation matrix $\Sigma$. The intervals given by equation 7 correct for simultaneous multiple comparisons made against one clone in particular (24). Further details are provided in the Appendix.

\section{RESULTS}

Identification of migrant aphid species in Pennsylvania snap bean fields. Surveys based on aphid trapping in six lateplanted commercial snap bean fields in Centre and Clinton Co., PA, were analyzed for species composition in 2004 to identify potential CMV vectors. A total of 25 aphid species were identified in trap catches and nine of these species occurred at greater than $2 \%$ of the total population (Table 1). Each of the remaining 16 species occurred rarely and each made up less than $1 \%$ of the total population. The most abundant species in 2004 was the corn leaf aphid, $R$. maidis, making up $22 \%$ of the trapped aphids. The next two most common aphids were the melon aphid, A. gossypii, and the yellow clover aphid, T. trifolii. Three other aphid species 
common to legume crops, A. fabae, A. glycines, and Acyrthosiphon pisum, occurred at 11,5 , and $5 \%$ of the population, respectively. No aphid species were observed colonizing snap bean plants in any of the six commercial bean fields during the July to August growing season. Lack of aphid colonization of snap bean was likely attributed to snap bean not being a preferred host for most of these species and the use of broad-spectrum insecticides targeting major insect pests (e.g., potato leafhopper, Empoasca fabae (Harris) and European corn borer, Ostrinia nubilalis Hübner). For these reasons, all alates observed in traps were presumed to be moving into snap bean fields from other hosts. Our findings were similar to those of Nault et al. (20) who reported a preponderance of $T$. trifolii, A. glycines, $R$. maidis, and A. pisum in New York snap bean fields. These dominant species were shown to vary from year to year and with planting date (20).

MVT tests. To identify potential aphid vectors transmitting CMV to snap bean and to compare relative transmission efficiency among aphid species, MVT tests were completed using transfers of 10 aphids per plant for inoculation probes. Results (Table 2), showing the number of plants infected (out of 50), indicated that 12 of 13 species tested were genetically competent to vector CMV-Le at different levels of efficiency. Empirical Bayesian estimates $\left(\hat{p}_{\mathrm{EB}}\right)$ of the probability of transmission by a single aphid of each clone are presented in Table 3.

The most effective vectors of CMV were A. gossypii, $A$. glycines, A. pisum, and T. trifolii ( $\hat{p}>0.05)$. Moderate vectors included A. spiraecola, $R$. maidis, A. craccivora, and $M$. euphorbiae $(0.01<\hat{p}<0.04)$. The poorest vectors $(\hat{p}<0.01)$ included two

TABLE 1. Dominant species in the aphid fauna trapped in snap bean fields in Pennsylvania a and New York ${ }^{\mathrm{b}}$

\begin{tabular}{ll}
\hline Aphid species $^{\mathrm{c}}$ & Common name \\
\hline Acyrthosiphon pisum (Harris) & Pea aphid \\
Aphis craccivora Koch & Cowpea aphid \\
Aphis fabae Scopoli & Black bean aphid \\
Aphis glycines Matsumura & Soybean aphid \\
Aphis gossypii Glover & Melon/cotton aphid \\
Myzus persicae (Sulzer) & Green peach aphid \\
Rhopalosiphum maidis (Fitch) & Corn leaf aphid \\
Rhopalosiphum padi (L.) & Bird cherry-oat aphid \\
Therioaphis trifolii (Monell) & Yellow clover aphid \\
\hline
\end{tabular}

a Based on surveys reported in this paper.

b Based on surveys by Nault et al. (20).

${ }^{\mathrm{c}}$ Following Blackman and Eastop (3). legume-feeding aphids, the clover aphid ( $N$. bakeri) and the black bean aphid (A. fabae), as well as the green peach aphid (M. persicae), which is an effective vector of many other plant viruses (3). Only one species, the English grain aphid (S. avenae) failed to transmit CMV-Le (Table 3). The HPD intervals show that A. gossypii, A. glycines, and T. trifolii may have transmission efficiencies above $10 \%$.

The point estimates and credible intervals for $p$ strongly indicate that $A$. glycines is a relatively efficient vector of CMV-Le when compared with other aphid species common to snap bean in $\mathrm{NY}$ and PA. We calculated $95 \%$ simultaneous confidence intervals for $\Psi_{i}$, the difference in transmission efficiency between clone $i$ and the $A$. glycines (PA) clone (Table 3 ). The intervals show that A. glycines is clearly as efficient a vector of CMV-Le as $A$. gossypii, A. pisum, and T. trifolii, and is a significantly better vector than any of the remaining clones tested.

The results also indicate consistency of transmission efficiency among different populations within a species. A clone of $A$. gossypii collected from cucumber in NY (A. gossypii $[\mathrm{NY}])$ and a clone collected from cotton in CA (A. gossypii [CA]) were both observed to transmit CMV-Le effectively (Table 3 ). In contrast, geographically distinct clones of A. fabae collected from two different locations in PA were both ineffective vectors of CMVLe. Two closely related small grain feeding Rhopalosiphum species differed in CMV transmission ability, with $R$. maidis a moderately effective vector and $R$. padi transmitting rarely.

SVT tests. SVT tests are a direct determination of transmission efficiency by single aphids. In three replicated tests of 120 aphids each, single aphids were transferred individually to snap bean seedlings for the inoculation probing access period. Three weeks later plants were ELISA-tested to verify infection. Results (Table 4) show the number of plants infected by single aphids from clones of four aphid species. The aphid clones tested represent high, moderate, and low efficiency vectors based on the previous MVT tests. The data indicate reasonable consistency in clonal transmission efficiency across experiments. Table 5 presents the point estimates for transmission efficiency by a single aphid and the $95 \%$ associated Agresti-Coull confidence intervals. The two A. glycines clones were the most efficient transmitters of CMV-Le among the five clones tested. We were interested in how much better (or worse) the A. glycines (PA) clone was, in comparison to the other clones, in transmitting CMV-Le. Table 5 shows the estimated $95 \%$ confidence intervals for $\delta_{i}$. The A. glycines (NY) and $A$. gossypii (NY) clones are not statistically different from the

TABLE 2. Total number and percentage of snap bean seedlings infected with Cucumber mosaic virus (CMV) in multiple vector transmission tests of 13 aphid species ( 15 clones)

\begin{tabular}{|c|c|c|c|c|c|c|c|}
\hline \multirow[b]{2}{*}{ Aphid clone } & \multicolumn{5}{|c|}{ Replicate $^{\mathrm{a}}$} & \multirow[b]{2}{*}{ Total } & \multirow[b]{2}{*}{$\%$} \\
\hline & 1 & 2 & 3 & 4 & 5 & & \\
\hline Aphis gossypii (NY) & 9 & 9 & 8 & 7 & 5 & 38 & 76 \\
\hline Therioaphis trifolii (PA) & 7 & 5 & 5 & 7 & 5 & 29 & 58 \\
\hline Acyrthosiphon pisum (PA) & 3 & 4 & 8 & 2 & 4 & 21 & 42 \\
\hline Aphis spiraecola $(\mathrm{PA})$ & 2 & 4 & 1 & 2 & 6 & 15 & 30 \\
\hline Macrosiphum euphorbiae (PA) & 3 & 2 & 0 & 0 & 3 & 8 & 16 \\
\hline Nearctaphis bakeri (PA) & 2 & 1 & 0 & 1 & 0 & 4 & 8 \\
\hline Myzus persicae (PA) & 1 & 0 & 1 & 0 & 1 & 3 & 6 \\
\hline Rhopalosiphum padi (NY) & 0 & 1 & 1 & 0 & 1 & 3 & 6 \\
\hline Aphis fabae (PA Adams Co.) & 0 & 1 & 0 & 0 & 0 & 1 & 2 \\
\hline Aphis fabae (PA Centre Co.) & 0 & 0 & 1 & 0 & 0 & 1 & 2 \\
\hline Sitobion avenae $(\mathrm{NY})$ & 0 & 0 & 0 & 0 & 0 & 0 & 0 \\
\hline
\end{tabular}

a Each replicate consisted of ten 10-day-old bean seedlings each of which was infested with 10 aphids previously allowed a $24 \mathrm{~h}$ unrestricted acquisition access and inoculation probing period on source tissue infected with CMV-Le. The column values are the number of infested plants out of 10 which became infected with CMV. As negative controls, 50 aphids of each clone were given a combined unrestricted acquisition access and inoculation probing period on healthy bean tissue as the source and on 10-day-old test seedlings for inoculation. Five seedlings were infested for each clone in each of 5 replicated treatments. None of 25 plants infested with a total of approximately 1,250 aphids of each clone became infected. 
A. glycines (PA) clone in CMV-Le transmission efficiency. The $R$. maidis (PA) and $M$. persicae (PA) clones are significantly worse CMV-Le transmitters than the A. glycines (PA) clone. The estimated transmission probabilities and the relative ranking of species for vector efficiency were similar between MVT and SVT tests (Tables 3 and 5). The SVT results independently verified that transmission efficiency estimates based on the MVT protocol (Table 3) accurately reflected the probability of single aphid transmission efficiency.

Comparison of legume-infecting CMV isolates by MVT tests. To test for the consistency of vector efficiency estimates when using different isolates of a virus, two aphid species were tested for their ability to transmit three isolates of CMV obtained from different sources. All three isolates were originally isolated from snap bean and, previous to this work, had been maintained in snap bean by sequential mechanical inoculations. All three isolates were aphid-transmitted to snap bean by $M$. persicae in preliminary multivector transmissions to maintain the isolates (data not shown). In positive control treatments using 50 aphids per plant, both $A$. glycines and $M$. persicae transmitted each CMV isolate to $100 \%$ ( 9 of 9) of the plants infested. While the positive control results show that both aphid species are genetically competent to vector all three CMV isolates, the fact that all plants in the positive controls tested positive in the MVT procedure using large numbers of aphids does not allow for the estimation of CMV transmission efficiency by a single aphid. Single aphid CMV transmission efficiencies can be estimated, however, from results of the main trial, in which groups of 10 aphids each were transferred to plants. Aphid vector efficiency was consistent for all three CMV isolates for a given species. In three replicated experiments using 10 aphids per plant, the percentage of 45 plants becoming infected with CMV-Le, CMV-Hy, and CMV-B when probed by $M$. persicae was $8.8,6.7$, and $0 \%$, respectively. In parallel treatments with A. glycines, the percentage of 45 plants becoming infected with CMV-Le, CMV-HY, and CMV-B was $68.8,73.3$, and $64.4 \%$, respectively. Table 6 shows the empirical Bayesian point estimates and credible intervals for the transmission efficiencies of each of the isolates by a single aphid. It is apparent that $M$. persicae was an inefficient vector of all three CMV isolates. For $M$. persicae, the credible intervals overlap, indicating no preferential transmission of any of the three CMV isolates. The same holds true for transmission by A. glycines. One can also see (Table 6) that A. glycines is more efficient in vectoring CMV than $M$. persicae. Simultaneous $95 \%$ confidence intervals were constructed for the differences in transmission efficiency when compared with the transmission of CMV-Le by the A. glycines (PA) clone (Table 6). The A. glycines (PA) clone is statistically more efficient in transmitting CMV-Le than $M$. persicae is in transmitting any of the three CMV isolates. All three CMV isolates do not differ statistically in their ability to be transmitted by the A. glycines (PA) clone. These results verify that aphid species transmission efficiency is consistent among the three different legume-infecting CMV isolates tested.

We also calculated the frequentist estimates $\hat{p}_{\mathrm{MLE}}$ and $\hat{p}_{\mathrm{B}}$ and associated confidence intervals for all the MVT data. These were similar to the empirical Bayesian estimates (data not shown).

\section{DISCUSSION}

This is the first comprehensive study on CMV transmission efficiencies of various aphid species that have been implicated in the spread of CMV into snap bean fields. Our approach allowed

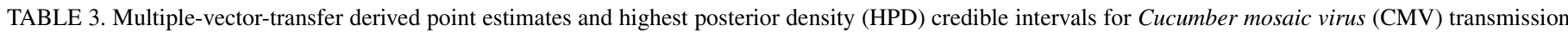

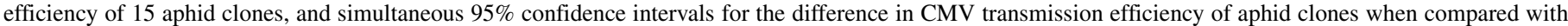
the Aphis glycines (PA) clone

\begin{tabular}{|c|c|c|c|c|c|}
\hline Clone & $\hat{p}_{\mathrm{EB}}{ }^{\mathrm{a}}$ & $\hat{\mathrm{L}}_{\mathrm{HPD}}{ }^{\mathrm{b}}$ & $\hat{\mathrm{U}}_{\mathrm{HPD}}$ & $\hat{\psi}_{L}{ }^{c}$ & $\hat{\psi}_{\mathrm{U}}$ \\
\hline Aphis gossypii (NY) & 0.1310 & 0.0925 & 0.1762 & -0.0303 & 0.1211 \\
\hline Aphis gossypii (CA) & 0.1062 & 0.0735 & 0.1439 & -0.0480 & 0.0883 \\
\hline Aphis glycines (PA) & 0.0865 & 0.0582 & 0.1185 & $\ldots$ & $\ldots$ \\
\hline Therioaphis trifolii (PA) & 0.0821 & 0.0548 & 0.1129 & -0.0662 & 0.0572 \\
\hline Acyrthosiphon pisum (PA) & 0.0524 & 0.0322 & 0.0752 & -0.0893 & 0.0202 \\
\hline Aphis spiraecola $(\mathrm{PA})$ & 0.0347 & 0.0190 & 0.0522 & -0.1035 & -0.0015 \\
\hline Rhopalosiphum maidis (PA) & 0.0347 & 0.0190 & 0.0522 & -0.1035 & -0.0015 \\
\hline Aphis craccivora $(\mathrm{MD})$ & 0.0268 & 0.0134 & 0.0418 & -0.1099 & -0.0110 \\
\hline Macrosiphum euphorbiae (PA) & 0.0171 & 0.0070 & 0.0286 & -0.1178 & -0.0227 \\
\hline Nearctaphis bakeri (PA) & 0.0082 & 0.0020 & 0.0156 & -0.1252 & -0.0333 \\
\hline Myzus persicae (PA) & 0.0061 & 0.0011 & 0.0122 & -0.1269 & -0.0359 \\
\hline Rhopalosiphum padi (NY) & 0.0061 & 0.0011 & 0.0122 & -0.1269 & -0.0359 \\
\hline Aphis fabae (PA Adams Co.) & 0.0020 & 0.0000 & 0.0048 & -0.1303 & -0.0408 \\
\hline Aphis fabae (PA Centre Co.) & 0.0020 & 0.0000 & 0.0048 & -0.1303 & -0.0408 \\
\hline Sitobion avenae $(\mathrm{NY})$ & 0.0010 & 0.0000 & 0.0026 & -0.1320 & -0.0431 \\
\hline
\end{tabular}

a $\hat{p}_{\mathrm{EB}}=$ the empirical Bayesian estimate of $p$, the probability of CMV-Le transmission by a single aphid

b $\hat{\mathrm{L}}_{\mathrm{HPD}}=$ lower limit, $\hat{\mathrm{U}}_{\mathrm{HPD}}=$ upper limit estimates of the HPD credible interval for $p$.

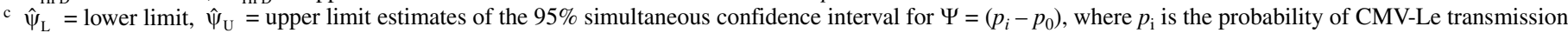
by a single aphid of clone $i$, and $p_{0}$ is the transmission efficiency of CMV-Le by a single aphid of the A. glycines (PA) clone.

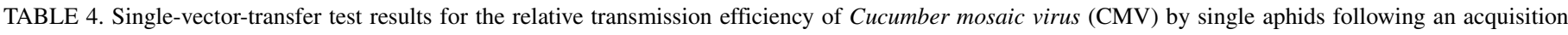
from infected snap bean

\begin{tabular}{|c|c|c|c|c|c|}
\hline \multirow[b]{2}{*}{ Aphid species } & \multicolumn{3}{|c|}{ Experiment $^{\mathrm{a}}$} & \multirow[b]{2}{*}{ Total $^{\mathrm{b}}$} & \multirow[b]{2}{*}{$\%$} \\
\hline & 1 & 2 & 3 & & \\
\hline Aphis glycines (NY) & 7 & 25 & 12 & 44 & 12.2 \\
\hline Aphis glycines (PA) & 9 & 14 & 15 & 38 & 10.6 \\
\hline Aphis gossypii $(\mathrm{NY})$ & 10 & 12 & 13 & 35 & 9.7 \\
\hline Rhopalosiphum maidis (PA) & 3 & 5 & 3 & 11 & 3.1 \\
\hline Myzus persicae $(\mathrm{PA})$ & 1 & 1 & 1 & 3 & 0.8 \\
\hline
\end{tabular}

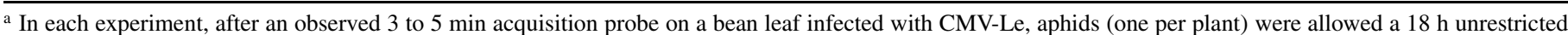
inoculation period on 10-day-old seedlings of cvs. Hystyle or Zeus. There were 120 replicate plants per experiment. For the controls, none of the 20 plants became infected when probed by 200 aphids of each species after they were first fed on healthy snap bean.

b Total number of plants becoming infected by CMV out of 360 aphid-infested plants. 
for the estimation of CMV transmission by a single aphid of a given clone, for the statistical comparison of transmission efficiencies across different species (or clones), and among different legume-infecting isolates of CMV. Four of 13 aphid species tested were identified as efficient vectors of CMV and all four were major components of aphid populations trapped in snap bean fields. These four species (A. gossypii, A. glycines, T. trifolii, and A. pisum) were migrating through snap bean fields, but none colonized the crop. In some years, a fifth aphid vector, $R$. maidis was detected in large numbers presumably migrating into snap bean fields from nearby maize crops. The aphid species predominating in the snap bean fields varied from year to year and during different times of the year (20). Therefore, the importance of any of the above species in CMV epidemics in snap bean may vary based on species composition of the migrant aphid population. Species composition is in turn influenced by composition of adjacent crops, planting dates, which can impact plant age at time of infection, and the effect of environmental factors on aphid behavior.

The role of A. glycines, A. gossypii, A. pisum, M. persicae, and T. trifolii as vectors of several nonpersistently transmitted viruses infecting legume field crops is well documented $(8,38)$. Studies by Doolittle (9) and Jagger (17) on transmission of cucumber mosaic disease by A. gossypii were among the first published reports of aphid transmission of any pathogenic agent. Since then, $M$. persicae and $A$. gossypii have been used extensively in molecular studies of the CMV aphid transmission mechanism using common strains of CMV infecting nonlegume hosts (23). However, less is known about the ability of aphid species to specifically transmit legume isolates of CMV. Early studies of CMV in legumes documented the ability of $M$. persicae to transmit CMV to beans (39). As a result of the recent population explosions of $A$. glycines on soybean in the United States, much effort has been directed toward studying its ability to transmit viruses. Although A. glycines is known to colonize only soybean during the growing season (26), large populations of alates emigrate from soybean to other crops $(5,20)$. Because A. glycines does not survive for long on legume species other than soybean, individual aphids tend to remain unsettled and move about probing potential hosts. This activity involving multiple probes and plant-to-plant movement enhances the opportunity for nonpersistent virus transmission by $A$. glycines. In addition to CMV (this paper), A. glycines is an effective vector of Alfalfa mosaic virus, Bean yellow mosaic virus, Soybean mosaic virus, Tobacco etch virus, and Tobacco vein mottle virus $(13,38)$.

At present, the reservoir of hosts required for CMV to survive between annual snap bean crops is unknown. Legume strains of CMV are not readily seed transmitted $(25,39)$, and our tests of 1,200 seedlings grown from seed collected from greenhouse grown CMV-Le infected plants were negative (data not shown). However, this source of virus can never be completely negated. Although soybean-infecting strains of CMV occur (14), none of the three strains of CMV used in this study were able to infect soybean (data not shown) and significant spread of CMV in soybean has not been reported to occur in the United States. Perennial alfalfa fields are known to harbor CMV and span over a half a million acres in snap bean-producing areas in the Northeast. A recent study in NY (29) showed that CMV levels in snap bean fields adjacent to CMV-infected alfalfa fields were identical to CMV levels in snap bean fields over 1 mile away from alfalfa. These results may indicate that aphids did not acquire CMV from adjacent alfalfa crops before transmitting it into snap bean fields. Alternatively, aphids may still acquire CMV from alfalfa, but their subsequent dispersal to snap bean fields is not limited to adjacent fields. More research is needed to elucidate the impact of alfalfa and other CMV reservoirs on the epidemiology of CMV in snap bean fields.

Knowledge of vector transmission efficiency is critical from an epidemiological perspective for complete understanding of pathogen spread and for developing disease management models and disease control strategies. SVT tests to determine vector efficiency require large numbers of replicated treatments, and are generally time consuming and labor intensive. Our results support the use of the less labor intensive MVT tests for estimating vector efficiency when combined with appropriate statistical analysis. The validity of these estimates depends on several independent factors. For instance, the estimation of $p$ (probability of transmission by a single aphid) from the results of MVT tests depends

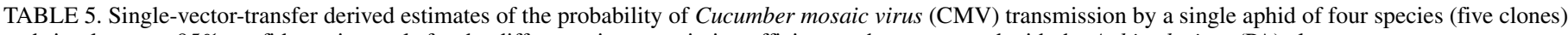
and simultaneous $95 \%$ confidence intervals for the difference in transmission efficiency when compared with the Aphis glycines (PA) clone

\begin{tabular}{|c|c|c|c|c|c|}
\hline Clone & $\hat{p}^{\mathrm{a}}$ & $\hat{\mathrm{L}}_{\mathrm{AC}}{ }^{\mathrm{b}}$ & $\hat{\mathrm{U}}_{\mathrm{AC}}$ & $\hat{\delta}_{L}{ }^{c}$ & $\hat{\delta}_{\mathrm{U}}$ \\
\hline Aphis glycines (NY) & 0.1222 & 0.0921 & 0.1603 & -0.0399 & 0.0732 \\
\hline Aphis glycines (PA) & 0.1056 & 0.0776 & 0.1418 & $\ldots$ & $\ldots$ \\
\hline Aphis gossypii $(\mathrm{NY})$ & 0.0972 & 0.0704 & 0.1325 & -0.0621 & 0.0454 \\
\hline Rhopalosiphum maidis (PA) & 0.0306 & 0.0165 & 0.0545 & -0.1193 & -0.0307 \\
\hline Myzus persicae (PA) & 0.0083 & 0.0017 & 0.0254 & -0.1376 & -0.0569 \\
\hline
\end{tabular}

a Estimate of $p$, the probability of CMV-Le transmission by a single aphid. A total of 360 individual single vector transfer tests were done for each clone.

${ }^{\mathrm{b}}$ Lower $\left(\hat{\mathrm{L}}_{\mathrm{AC}}\right.$ ) and upper ( $\hat{\mathrm{U}}_{\mathrm{AC}}$ ) limit estimates of the $95 \%$ Agresti-Coull intervals for $p$.

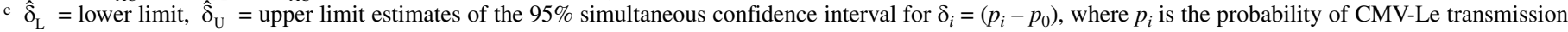
by a single aphid of clone $i$, and $p_{0}$ is the transmission efficiency of CMV-Le by a single aphid of the A. glycines (PA) clone.

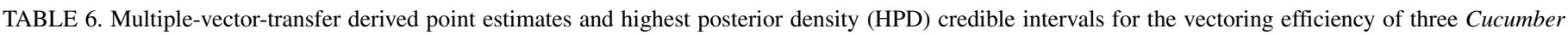

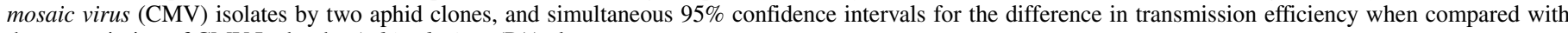
the transmission of CMV-Le by the Aphis glycines (PA) clone

\begin{tabular}{|c|c|c|c|c|c|c|}
\hline Clone & Isolate & $\hat{p}_{\mathrm{EB}}{ }^{\mathrm{a}}$ & $\hat{\mathrm{L}}_{\mathrm{HPD}}{ }^{\mathrm{b}}$ & $\hat{\mathrm{U}}_{\mathrm{HPD}}$ & $\hat{\psi}_{\mathrm{L}}{ }^{\mathrm{c}}$ & $\hat{\psi}_{U}$ \\
\hline \multirow[t]{3}{*}{ M. persicae (PA) } & CMV-Le & 0.0092 & 0.0023 & 0.0174 & -0.1518 & -0.0500 \\
\hline & CMV-Hy & 0.0068 & 0.0012 & 0.0136 & -0.1539 & -0.0528 \\
\hline & CMV-B & 0.0011 & 0.0000 & 0.0029 & -0.1598 & -0.0606 \\
\hline \multirow[t]{3}{*}{ A. glycines (PA) } & CMV-Le & 0.1085 & 0.0737 & 0.1491 & $\ldots$ & $\ldots$ \\
\hline & CMV-Hy & 0.1218 & 0.0837 & 0.1666 & -0.0600 & 0.0872 \\
\hline & CMV-B & 0.0968 & 0.0648 & 0.1338 & -0.0792 & 0.0553 \\
\hline
\end{tabular}

\footnotetext{
a $\hat{p}_{\mathrm{EB}}=$ the empirical Bayesian estimate of $p$, the probability of CMV transmission by a single aphid.

b $\hat{\mathrm{L}}_{\mathrm{HPD}}=$ lower limit, $\hat{\mathrm{U}}_{\mathrm{HPD}}=$ upper limit estimates of the HPD credible interval for $p$.

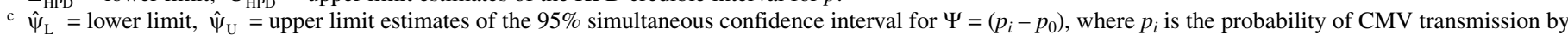
a single aphid of clone $i$, and $p_{0}$ is the transmission efficiency of CMV-Le by a single aphid of the A. glycines (PA) clone.
} 
on the assumption that $T$ (the number of test plants out of $n$ which become infected) has a binomial distribution. If the distribution of $T$ is not binomial, then the probability framework which allows one to estimate $p$ and its confidence intervals from MVT test results is not valid. Both Swallow (31) and Tebbs and Bilder (33) discuss the biological and statistical assumptions that are inherent in the MVT situation. The latter (33) list five assumptions which should be met for the assumption of the binomial distribution for $T$ to hold, and they are repeated here: (i) the status of each aphid vector (i.e., whether it carries the virus or not) is an independent and identically distributed Bernoulli random variable with viruspositive probability $p, 0<p<1$; (ii) aphids are assigned to plants at random; (iii) each aphid in the group ( $s$ per group) transmits the virus independently of $s$; (iv) the test plants do not differ in their resistance response to virus infection; (v) test plants are not misclassified. It can be challenging to verify these assumptions empirically. An indirect gauge of whether assumptions (i) to (v) hold is to compare transmission efficiencies which have been estimated by both SVT and MVT for the same clone. CMV transmission efficiencies were estimated by both SVT and MVT for four clones: A. glycines (PA), A. gossypii (NY), M. persicae (PA), and $R$. maidis (PA). A comparison of the point estimates of $p$ derived from the SVT tests (Table 5) to the point estimates $\left(\hat{p}_{\mathrm{EB}}\right)$ derived from the MVT tests (Table 3 ) for these four clones shows that the differences between the two estimates is within \pm 0.04 , or $4 \%$. The results of the SVT and MVT tests are consistent.

Our analysis indicates that group or MVT testing can be used with appropriately derived confidence intervals as a reliable estimator of CMV transmission efficiency. This is important, because group testing is much less labor intensive, requires less time, supplies, and bench space; and, therefore, is less expensive. Results of our transmission tests were surprisingly consistent from test to test over a 2-year period, and when testing geographically different populations of aphid species. No significant differences were detected between clones of A. glycines, A. gossypii, or A. fabae collected from different locations. Especially surprising was the similarity of the A. gossypii clone collected from cucumber in NY to a clone originally collected from cotton in CA. Although these clones differed phenotypically in size and color, there was little difference in their ability to transmit CMV with relatively high efficiency. Likewise, vector efficiency was consistent when tested against three different snap bean-infecting isolates of CMV. These field isolates have not yet been sequenced, however, they were collected in different locations (WI and NY), or at different times (1974 versus 2004). The consistency of these observations suggests that these methods prevented environmental factors from influencing CMV transmission efficiency and that a genetically stable mechanism regulates vector efficiency of these bean-infecting isolates of CMV by the various aphid species tested.

\section{APPENDIX}

For $\mathrm{M}=4$, the multivariate standard normal distribution (MVN) with mean vector $\mu$ and correlation matrix $\Sigma$ is given by $\vec{X} \sim \operatorname{MVN}(\mu, \Sigma)$, where $\mu=\{0,0,0,0\}$ and

$$
\Sigma=\left(\begin{array}{cccc}
1 & \rho_{12} & \rho_{13} & \rho_{14} \\
\rho_{21} & 1 & \rho_{23} & \rho_{24} \\
\rho_{31} & \rho_{32} & 1 & \rho_{34} \\
\rho_{41} & \rho_{42} & \rho_{43} & 1
\end{array}\right)
$$

Note that $\Sigma$ is symmetric, as we have $\rho_{j k}=\rho_{k j}, j \neq k$ for the offdiagonal elements. The correlation parameters $\rho_{j k}=\omega_{j} \omega_{k}$ where

$$
\omega_{j}=\left(1+\frac{n_{0} \cdot p_{j}\left(1-p_{j}\right)}{n_{j} \cdot p_{0}\left(1-p_{0}\right)}\right)^{-1 / 2}
$$

One uses $\hat{\rho}_{j k}=\hat{\omega}_{j} \hat{\omega}_{k}$ as estimates of $\rho_{j k}$ in $\Sigma(24)$. For our data, $n_{0}=n_{j}=360, p_{0}=0.1056$ for A. glycines (PA), $p_{1}=0.1222$ for A. glycines (NY), $p_{2}=0.0972$ for A. gossypii (NY), $p_{3}=0.0083$ for $M$. persicae (PA), $p_{4}=0.0306$ for $R$. maidis (PA). Thus,

$$
\hat{\Sigma}=\left(\begin{array}{cccc}
1 & 0.493 & 0.656 & 0.597 \\
0.493 & 1 & 0.690 & 0.628 \\
0.656 & 0.690 & 1 & 0.837 \\
0.597 & 0.628 & 0.837 & 1
\end{array}\right)
$$

Critical values of $z_{\alpha, M, \Sigma}$ have been tabulated for constant $\rho_{j k}(10)$. With modern software, the critical value $\mathrm{z}_{\alpha, \mathrm{M}, \Sigma}$ given $\hat{\Sigma}$ above can be found by numerical integration. We did so with Mathematica (version 6, Wolfram Research, Inc., Champaign, IL), which returned $\mathrm{z}_{\alpha, \mathrm{M}, \Sigma}=2.38799$. The estimate was used in equation 7 to obtain the confidence intervals for $\delta_{i}$.

\section{ACKNOWLEDGMENTS}

This project was supported in part by a grant from the Pennsylvania Department of Agriculture (Grant No. ME 445583). The authors thank M. Bierly, Hanover Foods Inc., Center Hall, PA, for assistance in organizing grower cooperation and facilitating field studies.

\section{LITERATURE CITED}

1. Anderson, B. J., Boyce, P. M., and Blanchard, C. L. 1995. RNA 4 sequences from cucumber mosaic virus subgroups I and II. Gene 161:293-294.

2. Bilder, C. R., and Tebbs, J. M. 2005. Empirical Bayesian estimation of the disease transmission probability in multiple-vector-transfer designs. Biomet. J. 47:502-516.

3. Blackman, R. L., and Eastop, V. F. 2000. Aphids on the World's Crops: An Identification and Information Guide. 2nd Ed. John Wiley \& Sons, Inc., NY.

4. Brown, L. D., Cai, T. T., and DasGupta, A. 2001. Interval estimation for a binomial proportion. Stat. Sci. 16:101-117.

5. Burrows, M. E. L., Boerboom, C. M., Gaska, J. M., and Grau, C. R. 2005. The relationship between Aphis glycines and Soybean mosaic virus incidence in different pest management systems. Plant Dis. 89:926-934.

6. Burrows, P. M. 1987. Improved estimation of pathogen transmission rates by group testing. Phytopathology 77:363-365.

7. Chen, B., and Francki, R. I. B. 1990. Cucumovirus transmission by the aphid Myzus persicae is determined solely by the viral coat protein. J. Gen. Virol. 71:939-944.

8. Clark, A. J., and Perry, K. L. 2002. Transmissibility of field isolates of soybean viruses by Aphis glycines. Plant Dis. 86:1219-1222.

9. Doolittle, S. P. 1916. A new infectious mosaic disease of cucumber. Phytopathology 6:145-147.

10. Dunnett, C. W. 1955. A multiple comparison procedure for comparing several treatments with a control. J. Am. Stat. Assoc. 50:1096-1121.

11. Gibbs, A. J., and Gower, J. C. 1960. The use of a multiple-transfer method in plant virus transmission studies - some statistical points arising in the analysis of results. Ann. Appl. Biol. 48:75-83.

12. Gildow, F. E., Fleischer, S., Wiseman, M., Nault, B., and Shah, D. 2005. Aphid vectors associated with epidemics of cucumber mosaic virus (CMV) in snap beans. (Abstr.) Phytopathology 95(suppl.):S34.

13. Hill, J. H., Alleman, R., Hugg, D. B., and Grau, C. R. 2001. First report of transmission of Soybean mosaic virus and Alfalfa mosaic virus by Aphis glycines in the New World. Plant Dis. 85:561.

14. Hong, J. S., Ohnishi, S., Masuta, C., Choi, J. K., and Ryu, K. H. 2007. Infection of soybean by cucumber mosaic virus as determined by viral movement protein. Arch. Virol. 152:321-328.

15. Hsu, H. T., Barzuna, L., Hsu, Y. H., Bliss, W., and Perry, K. L. 2000. Identification and subgrouping of Cucumber mosaic virus with mouse monoclonal antibodies. Phytopathology 90:615-620.

16. Hung, M., and Swallow, W. H. 1999. Robustness of group testing in the estimation of proportions. Biometrics 55:231-237.

17. Jagger, I. C. 1916. Experiments with the cucumber mosaic disease. Phytopathology 6:148-151.

18. Larsen, R. C., Miklas, P. N., Eastwell, K. C., Grau, C. R., and Mondjana, A. 2002. A virus disease complex devastating late season snap bean production in the Midwest. Annu. Rep. Bean Improv. Coop. 45:36-37.

19. McCann, M. H., and Tebbs, J. M. 2007. Pairwise comparisons for proportions estimated by pooled testing. J. Stat. Plan. Infer. 137:12781290. 
20. Nault, B. A., Shah, D. A., Dillard, H. R., and McFaul, A. C. 2004. Seasonal and spatial dynamics of alate aphid dispersal in snap bean fields in proximity to alfalfa and implications for virus management. Environ. Entomol. 33:1593-1601.

21. Nault, L. R. 1997. Arthropod transmission of plant viruses: A new synthesis. Ann. Entomol. Soc. Am. 90:521-541.

22. Palukaitis, P., Roossinck, M. J., Dietzgen, R. G., and Francki, R. I. B. 1992. Cucumber mosaic virus. Adv. Virus Res. 41:281-348.

23. Perry, K. L., Zhang, L., and Palukaitis, P. 1998. Amino acid changes in the coat protein of cucumber mosaic virus differentially affect transmission by the aphids Myzus persicae and Aphis gossypii. Virology 242:204-210.

24. Piegorsch, W. W. 1991. Multiple comparisons for analyzing dichotomous response. Biometrics 47:45-52.

25. Provvidenti, R. 1976. Reaction of Phaseolus and Macroptilium species to a strain of cucumber mosaic virus. Plant Dis. Rep. 60:289-293.

26. Ragsdale, D. W., Voegtlin, D. J., and O’Neil, R. J. 2004. Soybean aphid biology in North America. Ann. Entomol. Soc. Am. 97:204-208.

27. Roossinck, M. J. 2002. Evolutionary history of Cucumber mosaic virus deduced by phylogenetic analyses. J. Virol. 76:3382-3387.

28. Sass, M., German, T., and Nienhuis, J. 2006. Assessing germplasm resistance to the soybean aphid virus complex. Annu. Rep. Bean Improv. Coop. 49:179-180.

29. Shah, D. A., Dillard, H. R., Mazumdar-Leighton, S., Gonsalves, D., and Nault, B. A. 2006. Incidence, spatial patterns, and associations among viruses in snap bean and alfalfa in New York. Plant Dis. 90:203210.

30. Smith, C. F., Eckel, R. W., and Lampert, E. 1992. A key to many of the common alate aphids of North Carolina (Aphididae:Homoptera). North Carolina Agriculture Research Service, Raleigh, NC.

31. Swallow, W. H. 1985. Group testing for estimating infection rates and probabilities of disease transmission. Phytopathology 75:882-889.

32. Taylor, A. G., and Shail, J. W. 2006. The impact of CMV in reducing yield of selected snap bean cultivars. Annu. Rep. Bean Improv. Coop. 49:199-200.

33. Tebbs, J. M., and Bilder, C. R. 2004. Confidence interval procedures for the probability of disease transmission in multiple-vector-transfer designs. J. Agric. Biol. Envir. S. 9:75-90.

34. Tebbs, J. M., Bilder, C. R., and Moser, B. K. 2003. An empirical Bayes group-testing approach to estimating small proportions. Commun. StatTheor. M. 32:983-995.

35. Tebbs, J. M., and Swallow, W. H. 2003. More powerful likelihood ratio tests for isotonic binomial proportions. Biomet. J. 45:618-630.

36. Turkkan, N., and Pham-Gia, T. 1997. AS 308 - Highest posterior density credible region and minimum area confidence region: The bivariate case. J. Roy. Stat. Soc. C. 46:131-140.

37. Wallis, C. M., Fleischer, S. J., Luster, D., and Gildow, F. E. 2005. Aphid (Hemiptera:Aphididae) species composition and potential aphid vectors of plum pox virus in Pennsylvania peach orchards. J. Econ. Entomol. 98:1441-1450.

38. Wang, R. Y., Kritzman, A., Hershman, D. E., and Ghabrial, S. A. 2006. Aphis glycines as a vector of persistently and nonpersistently transmitted viruses and potential risks for soybean and other crops. Plant Dis. 90:920926.

39. Whipple, O. C., and Walker, J. C. 1941. Strains of cucumber mosaic virus pathogenic on bean and pea. J. Agric. Res. 62:0027-0060. 